\title{
Extracellular Polysaccharides of Rhizobium Strains Associated with Lotus Species
}

\author{
By R. W. BAILEY, R. M. GREENWOOD AND A. CRAIG \\ Applied Biochemistry Division, D.S.I.R., Palmerston North, New Zealand
}

(Accepted for publication 8 January I97I)

\begin{abstract}
SUMMAR Y
The monosaccharide composition of extracellular polysaccharides isolated from IOI strains of rhizobia, principally associated with the lotus group of legumes, have been examined. Polysaccharides from 43 fast-growing, acidproducing strains ( 17 lotus) gave a substantially consistent monosaccharide pattern of glucose, uronic acid and galactose with little or no mannose. Polysaccharides from lucerne and goat's rue strains (four) of this class of rhizobia differed in that they contained almost no uronic acid. Fifty-eight slowgrowing, non-acid-producing strains ( 52 lotus) were also investigated. On centrifuging, 36 of them gave a mucilagenous clot of cells and polysaccharide and required dilute alkali treatment to separate the polysaccharide. No polysaccharide was produced by four non-acid-producing strains. Polysaccharides from the non-acid-producing rhizobia gave much more complex monosaccharide patterns. Glucose and mannose were nearly always present in large amounts; galactose and uronic acid were frequently absent while fucose, rhamnose and three unidentified sugars were occasionally present. Electron micrograph studies showed extracellular material which, in strains giving a clot, was not removed by centrifuging. Monosaccharide composition of the polysaccharides appeared to be correlated with the division into acidand non-acid-producing classes of rhizobia. Both classes of rhizobial strains associated with Lotus species conformed to this correlation apart from one strain which was intermediate.
\end{abstract}

\section{INTRODUCTION}

The monosaccharide compositions of the extracellular polysaccharides produced by a large number of rhizobial strains have been reported (see Graham, I965; Amarger, Obaton \& Blachère, 1967). Nearly all of the polysaccharides so described have, however, been isolated from fast-growing, acid-producing strains (Fred, Baldwin \& McCoy, 1932). With these rhizobia the diluted culture fluid is easily centrifuged to remove cells leaving polysaccharide in solution. In contrast, few polysaccharides produced by slow growing, non-acid-producing rhizobial strains have been examined. One reason for this may be that the polysaccharides from these strains are usually more difficult to isolate by diluting the cultures and centrifuging (Graham, 1965).

Norris (1965) showed that within the group of rhizobia nodulating Lotus species, both acid- and non-acid-producing strains occur, associated with different host species. We have been interested in the rhizobia associated with Lotus species growing in New Zealand, and an extensive collection of strains (both acid- and non-acidproducing) isolated from or able to nodulate these legumes has been built up by one of us (R.M.G.). Since published work on rhizobial polysaccharides includes few strains 
able to nodulate Lotus species the monosaccharide composition of the extracellular polysaccharides produced by a number of strains from our collection of lotus rhizobia have, therefore, been determined as part of a study of the classification of these bacteria. The majority of the organisms tested were non-acid-producing strains, many of them giving insoluble gums not easily separated from the bacteria. A simple alternative method for isolating these polysaccharides was, therefore, devised. To compare the results obtained in the present study with published results the monosaccharide composition of the extracellular polysaccharides from 32 Rhizobium strains, principally acid-producing, associated with genera other than the lotus group were also examined.

Organisms.

\section{METHODS}

Of IOI rhizobia isolates, 69 ( 17 acid-producers and 52 non-acid-producers) nodulated Lotus species. There were nine isolates of clover (Trifolium spp.) rhizobia, two isolates each of lucerne (Medicago sativa L.), pea vetch (Pisum, Vicia), garden bean (Phaseolus vulgaris L.), goat's rue (Galega officinalis L.), Sophora tomentosa L., peanut (Arachis hypogaea L.) and chick pea (Cicer arietinum L.) rhizobia; one isolate each from Acacia dealbata Link., crown vetch (Coronilla varia L.), cowpea (Vigna) and six isolates from New Zealand indigenous legumes (Carmichaelia and Sophora spp.). Strain numbers of these organisms are given in Tables I, 2, and 3. All isolations were made at Palmerston North except for the following organisms. Strains CB 562, СB 569, СB 745, СB 756, CB 782, СВ935, СВ II89 and CB II 99 were from Dr D. O. Norris, C.S.I.R.O., Brisbane, Queensland, Australia. Strains CC809a, CC 8I I, CC8I4s, CC 856 and CC I005 were from Mr J. Brockwell, C.S.I.R.O., Canberra, Australia. Strains SU 202, TA I, wU95 and wU 425 were from Dr C. A. Parker, University of Western Australia, Perth. Strains SU 343, SU 39I and CC5II were from Dr R. A. Date, U-DALS, New South Wales, Australia. Strain 3G I6I was from Dr U. M. Means, U.S.D.A., Beltsville, Maryland, U.S.A. Strains PDD lucerne (NZP 4008), SU 47, I346 (NZP 5087), I3I4 (NZP 5088), w 72 (NZP 525I) and CC829 (NZP 202I) were from Mr A. Hastings, D.S.I.R., Auckland, New Zealand, and birdsfoot trefoil 46I Canada strain (NZP 2227) and F.J.N. (NZP 2I 54) were from Mr G. G. Taylor, Fruitgrowers Chemical Co. Ltd, Nelson, New Zealand.

All strains were checked for freedom from contamination and ability to nodulate host plants. Strains were maintained on slopes of yeast mannitol agar medium (Graham, 1965). For polysaccharide production the organisms were grown in the same medium (200 ml.) without agar in conical flasks (I 1.) for Io days at $28^{\circ}$ in a Gallenkamp orbital incubator.

Isolation of extracellular polysaccharide. Cultures ( $150 \mathrm{ml}$.) diluted with an equal volume of water were centrifuged at $3000 \mathrm{~g}$ for $30 \mathrm{~min}$. and the cell-free solution decanted from the pellet. Ethanol ( 3 vol.) was added to the supernatant with stirring and, after standing overnight at $2^{\circ}$, the precipitated polysaccharide was harvested by centrifuging, dissolved in water (100 $\mathrm{ml}$.), dialysed for 2 to 3 days against tap water and freeze-dried. While this procedure was satisfactory with all of the acid-producing and some of the non-acid-producing strains, many of the latter strains gave on dilution and centrifuging a large viscous clot, with the bacteria dispersed through it, filling about half the liquid volume. The following modification was therefore used with cultures of all non-acid-producing strains which on a preliminary centrifuging of culture $(50 \mathrm{ml}$.) formed this clot. Undiluted culture fluid (I $50 \mathrm{ml}$.) was made approximately $0.5 \mathrm{~N}$ with 
IO N-NaOH ( 7 to $8 \mathrm{ml}$.), stirred for $30 \mathrm{~min}$., centrifuged to remove organisms and immediately acidified with $50 \%(\mathrm{v} / \mathrm{v})$ acetic acid. Polysaccharides were not precipitated on acidification but still required ethanol (3 vol.) for precipitation. As isolated, the polysaccharides contained 70 to $80 \%$ carbohydrate (anthrone + carbazole values) and were not further purified.

Carbohydrate analyses. Total neutral hexoses and methyl pentoses in the polysaccharides were measured by an anthrone method (Bailey, 1967) using a glucose standard. Total uronic acids were measured by the carbazole-sulphuric acid method of Dische as described by Montreuil \& Spik (1963) using a glucuronic acid standard. Control experiments showed that pyruvate (Dudman \& Heidelberger, I969) did not interfere in this reaction. Neutral hexoses interfere slightly in the carbazole reaction and a correction was therefore applied to each uronic acid value. This correction was calculated from a glucose-carbazole standard and the neutral hexose value obtained by anthrone for each polysaccharide. No attempt was made to differentiate between glucuronic acid and 4-O-methyl glucuronic acid both of which occur in these polysaccharides (Humphrey \& Vincent, 1959; Graham, 1965). As in most other studies of rhizobial polysaccharides the neutral monosaccharide portion of any acid-resistant aldobiouronic acid liberated during hydrolysis was not identified.

Individual neutral monosaccharides present in polysaccharide hydrolysates were measured by quantitative paper chromatography (Wilson, 1959) using acid-washed paper and aniline hydrogen phosphate spray reagent. Two samples of each polysaccharide were usually hydrolysed. Results were calculated against the appropriate monosaccharide standards except for $\mathrm{X}_{1}, \mathrm{X}_{2}$ and $\mathrm{X}_{3}$ which were calculated as glucose. Polysaccharides (IO mg.) were hydrolysed with I N-H $\mathrm{SO}_{4}\left(2 \mathrm{ml}\right.$.) for $4 \mathrm{~h}$. at $100^{\circ}$. The hydrolysates were neutralized with $\mathrm{BaCO}_{3}$, filtered and concentrated to a small volume (I ml.). Chromatograms were developed with either solvent (I) ethyl acetate + water + pyridine $(\mathrm{I} 2+4+5$, by vol.) or (2) $n$-butyl acetate + water + ethanol + pyridine $(8+\mathrm{I}+$ $2+2$, by vol.). Both of these solvents clearly separate glucose, galactose and mannose from one another in mixtures, uronic acids remain at the starting line and uronic acid lactones run off the paper. Other solvents used were (3) ethyl acetate + acetic acid + formic acid + water $(18+3+\mathrm{I}+4$, by vol.), and (4) $n$-butanol +ethanol + water $(4+I \cdot I+I \cdot 9$, by vol.). Details of spray reagents (aniline hydrogen phosphate, $p$ anisidine $\mathrm{HCl}$, naphthoresorcinol, ninhydrin, thiobarbiturate and diphenylamine aniline) were given by Bailey (1969).

Electron microscopy. Bacterial suspensions were sprayed onto carbon-coated collodion films, shadowed with platinum off carbon electrodes and examined in a Philips EM 200 electron microscope. Bacterial suspensions were prepared from ro day cultures or from isolated bacteria. Cultures were diluted $\mathrm{I}: \mathrm{ro}$ in order to minimize the effect of salts and used directly. Bacteria were centrifuged ( $3000 \mathrm{~g}$ for $30 \mathrm{~min}$.) from acidproducing cultures after diluting $\mathrm{I}: \mathrm{I}$ or from non-acid-producing cultures after alkali treatment and resuspended in the same amount of distilled water as the original culture. Cultures of non-acid-producing strains containing clots were also centrifuged directly and culture fluid decanted from the clot which was then redispersed in distilled water to its original volume. 


\section{RESULTS}

Extracellular polysaccharides from acid-producing rhizobia

The neutral monosaccharide and uronic acid compositions of the extracellular polysaccharides produced by 43 strains of acid-producing rhizobia are given in Table I. They show a substantially similar pattern for the polysaccharides from rhizobia nodulating a wide range of host legumes. The only exceptions appear to be the lucerne and goat's rue rhizobia which produced polysaccharides differing mainly in their lack of uronic acid.

Table I. Monosaccharide composition of extracellular polysaccharides from acid-producing strains of rhizobia

\begin{tabular}{|c|c|c|c|c|c|c|}
\hline \multirow[b]{2}{*}{ Host legume and strain number } & \multirow{2}{*}{$\begin{array}{l}\text { Total } \\
\text { neutral } \\
\text { hexoses } \\
(\%)^{*}\end{array}$} & \multirow{2}{*}{$\begin{array}{l}\text { Yield } \\
\text { (g./1. of } \\
\text { culture) }\end{array}$} & \multicolumn{4}{|c|}{ Monosaccharides $(\%) \dagger$} \\
\hline & & & $\begin{array}{l}\text { Uronic } \\
\text { acid }\end{array}$ & $\begin{array}{c}\text { Galac- } \\
\text { tose }\end{array}$ & Glucose & Mannose \\
\hline Goat's rue NZP 5067, 5068 & 54 to 58 & $\mathrm{I} \cdot 5$ & 0 to 0.5 & 26 to 33 & 66 to $7 \mathrm{I}$ & o to $2 \cdot 7$ \\
\hline Lucerne NZP 4008 & 56 & $I \cdot I$ & - & $2 I$ & 75 & 4 \\
\hline Lucerne su 47 & 30 & 0.24 & $\mathbf{I}$ & I3 & 54 & 32 \\
\hline $\left.\begin{array}{l}\text { Carmichaelia spp. NZP 5061, 5083 } \\
\text { Sophora spp. NZP } 5055\end{array}\right\}$ & 46 to 65 & $I \cdot 6$ to $2 \cdot I$ & I5 to 30 & 16 to 33 & 49 to 65 & Trace \\
\hline $\left.\begin{array}{l}\text { Lotus spp. NZP } 2034,2037,2042, \\
2048,2079,2085,2093,2147 \\
2150,217 \text { x, su } 343, \operatorname{cc} 809 a\end{array}\right\}$ & 47 to 55 & $I \cdot 4$ to $2 \cdot 0$ & 20 to 30 & 21 to 24 & 50 to 60 & - \\
\hline $\begin{array}{l}\text { Lotus spp. NZP 2014, 2024, } 2077 \text {, } \\
\text { CC } 856,81 \text { I }\end{array}$ & 45 to 59 & $\mathrm{I} \cdot \mathrm{O}$ to $\mathrm{I} \cdot 5$ & 20 to 40 & I 8 to 22 & 40 to 60 & 2 to 5 \\
\hline $\begin{array}{l}\text { Chick pea CB I I 89, I199 } \\
\text { Clover NZP 514, 5039, 5098, 5107, } \\
\text { CB 782, SU 202, WU95, TA I } \\
\text { Bean NZP 5097 }\end{array}$ & 42 to 53 & 0.8 to $I \cdot 2$ & 23 to 35 & I3 to 14 & $5 \mathrm{I}$ to 57 & 7 to 9 \\
\hline $\begin{array}{l}\text { Pea vetch NZP 5225, SU 391 } \\
\text { Crown vetch 3G I6I } \\
\text { Sophora spp. NZP 5057 } \\
\text { Carmichaelia } \text { spp. NZP 5080, } \\
5105\end{array}$ & 42 to 52 & $\begin{array}{l}0.6 \text { to } 1 \cdot 0 \% \\
0.9 \text { to } 2 \cdot 8\end{array}$ & 30 to 40 & I5 to 28 & 34 to 45 & 2 to 5 \\
\hline Bean CC5II & 35 & 0.6 & 48 & 18 & 27 & 7 \\
\hline Clover NZP 5063 & 36 & 0.8 & $3 \mathrm{I}$ & 34 & 32 & 3 \\
\hline
\end{tabular}

* Percentage of neutral hexoses as anhydro-glucose by anthrone in freeze-dried polysaccharide corrected for moisture.

$\dagger$ Results calculated as percentage of the sum of the measured individual neutral monosaccharides in polysaccharide hydrolysate + corrected total uronic acid; - or $0=$ clearly absent.

$\ddagger$ Yields for clover strains only.

After Io days growth all of the strains gave cultures with low $\mathrm{pH}$ values $(4.0$ to $4.5)$ and all produced polysaccharide.

As a check on the use of alkali with cultures of non-acid-producing rhizobia, washed organisms from 20 cultures of the acid-producing strains were extracted with alkali and the extracts processed. Polymer yields were only 0.01 to $0.02 \mathrm{~g}$. from the organisms in I 1. of culture. Hydrolysates showed glucose and rhamnose (trace) on chromatograms in all cases and occasionally traces of galactose and mannose. There was never any sign of fucose or the unknowns $X_{1}, X_{2}$ or $X_{3}$ of Table 2. 
Table 2. Monosaccharide composition of extracellular polysaccharide from non-acid-producing strains of rhizobia

\begin{tabular}{|c|c|c|c|c|c|c|c|c|c|}
\hline \multirow[b]{2}{*}{ Host plant and strain numbers } & \multirow[b]{2}{*}{$\begin{array}{l}\text { Total neutral } \\
\text { hexoses }(\%) \\
\text { (as in Table I) }\end{array}$} & \multirow[b]{2}{*}{$\begin{array}{l}\text { Yield (g./1. of } \\
\text { culture) }\end{array}$} & \multicolumn{7}{|c|}{ Monosaccharides ( $\%$ as in Table 1 ) } \\
\hline & & & Uronic acid & Galactose & Glucose & Mannose & Fucose & $\begin{array}{l}\text { Rham- } \\
\text { nose }\end{array}$ & Unknown \\
\hline \multicolumn{10}{|c|}{ (a) Lotus strains } \\
\hline $\begin{array}{l}\text { NZP } 2076,2088,2100,2182,2257, * \\
5091,5144\end{array}$ & 32 to 55 & 0.3 to 0.7 & o to $\mathrm{I}$ & 16 to $2 I$ & 36 to 48 & 30 to 45 & 一 & - & - \\
\hline NZP $2075,{ }^{*} 5026, * 5274^{*}$ & I I to 42 & 0.1 I to 0.7 & - & 0 to 3 & o to 9 & 88 to 100 & - & - & - \\
\hline NZP $2170,2175,2240$ & 37 to 45 & 0.4 & 2 to 4 & 18 to 24 & 36 to 45 & 34 to 38 & - & - & - \\
\hline NZP $5059 / 2 *$ & 23 & 0.4 & 10 & 15 & 40 & 35 & 一 & - & - \\
\hline NZP $2243^{*}$ & 12 & 0.2 & 7 & - & 48 & 45 & - & - & - \\
\hline NZP $2227^{*}$ & 44 & 0.8 & 18 & 18 & 53 & II & - & - & - \\
\hline WU $425^{*}$ & 18 & 0.2 & 22 & 7 & 17 & 54 & - & - & - \\
\hline $\begin{array}{l}\text { NZP } 202 \text { If }, 2089,2180,2193,2200, \\
2254, \mathrm{CC} 814 \mathrm{~s}\end{array}$ & 40 to 56 & $0 \cdot 7-1 \cdot 4$ & 27 to 39 & - & 41 to 49 & 18 to 25 & - & - & - \\
\hline $\mathrm{NZP} 5042^{*}$ & 18 & 0.12 & 33 & - & $3 \mathrm{I}$ & 36 & - & - & - \\
\hline NZP 21 $86,2192,5052$ & $6 I$ to 68 & 0.7 to 1.0 & 21 to 23 & - & 36 to 39 & II to 14 & - & 25 to 32 & - \\
\hline NZP $2247, * 5025, * 5251 / 2 *$ & 23 to 38 & 0.2 to 0.3 & 0 to $2 \cdot 5$ & 31 to 35 & 26 to 30 & 30 to 40 & 3 to 6 & - & - \\
\hline NZP 5113 & 44 & 0.8 & - & 23 & 42 & 22 & I 2 & - & - \\
\hline $\begin{array}{l}\text { NZP 2087, } 2141,2151,2181,{ }^{*} 5032, \\
5033,5035,5044,5074,5087,5088^{*}\end{array}$ & 42 to 55 & 0.5 to 0.8 & 22 to 40 & 6 to 15 & 26 to 39 & 8 to 20 & - & - & 10 to $17 \mathrm{X}$ \\
\hline $\mathrm{NZP} 2128, * 2154,2183,2189$ & 37 to 51 & 0.2 to 0.6 & 16 to 25 & 20 to 40 & 19 to 28 & 12 to 28 & - & - & 5 to $19 X$ \\
\hline NZP 2073* & 28 & 0.6 & I.5 & 36 & 27 & 33 & - & - & $2 \cdot 5 X_{2}$ \\
\hline \multicolumn{10}{|c|}{ (b) Non-Lotus strains } \\
\hline Peanut Св935* & $4 r$ & 0.4 & 0.5 & $4 I$ & $30 \cdot 5$ & 23 & - & 5 & - \\
\hline Peanut CB 745 & 42 & - & 23 & 7 & 45 & 15 & - & - & $10 X_{1}$ \\
\hline Acacia dealbata NZP 5073 & 53 & 0.6 & 26 & - & 35 & I4 & - & 25 & - \\
\hline Sophora tomentosa СВ 569 & II & 0.3 & $2 \cdot 5$ & - & 一 & 9 & - & - & - \\
\hline Sophora tomentosa Св $562 *$ & 25 & 0.2 & 18 & - & 一 & 32 & - & 50 & - \\
\hline Cowpea CB 756 & 54 & 0.4 & 20 & 9 & 37 & 20 & I & - & $13 X_{3}$ \\
\hline
\end{tabular}

* Cultures did not give typical mucilagenous clot on centrifuging; alkali not used in isolation. 


\section{Extracellular polysaccharides from non-acid-producing rhizobia}

The neutral monosaccharide and uronic acid composition of the polysaccharides isolated from cultures of 54 strains of non-acid-producing rhizobia, 48 of which were associated with Lotus species, are given in Table 2 together with an indication of clot production. Four strains (NZP 222 I, 2245, 2246; CC 1005) gave no clot or extracellular polysaccharide. There was a wide variation possible in polysaccharide composition which appeared to extend beyond the Lotus group. Several consistencies in monosaccharide composition are evident. All 12 polymers containing unknown $\mathrm{X}_{1}$ have a common monosaccharide composition as does one group of seven galactose-free polymers. High mannose content generally correlates with very low uronic acid levels while high uronic acid levels correlate with lower amounts of mannose. Four out of six polymers containing rhamnose also have a similar monosaccharide pattern. The fact that 26 of the 54 polysaccharides from non-acid-producing rhizobia are entirely free from galactose or uronic acid suggests that if these polysaccharides are mixtures at least the mixtures do not contain any of the usual polysaccharide produced by most acid-producing rhizobia.

After Io days growth these non-acid-producing organisms gave cultures within the $\mathrm{pH}$ range 5.5 to 7.5 which generally contained less polysaccharide than the acidproducing ones. Polysaccharides isolated with alkali from clot-producing cultures appeared to be more difficult to redissolve in water than those from acid-producing rhizobia. The anthrone values suggested that occasionally these crude preparations contained very little actual polysaccharide and these cases were nearly always ones of very low yields of material.

The possibility existed that alkali was extracting an additional or different polysaccharide, which might explain some of the variations listed in Table 2. Polymer extracted by alkali from six strains of bacteria yielding no clot was therefore examined, including two which gave no polysaccharide in their culture fluid. In five cases (NZP 222I, 2227, 5059/2, 525I/2 and CC 1005) alkali extraction did not contribute additional polysaccharide. With strain (NZP 5274), however, alkali treatment extracted a polysaccharide whose composition (high galactose, glucose, mannose and rhamnose but no uronic acid) clearly differed from the composition of the polysaccharide in the cell-free culture fluid (Table 2). In some cases, therefore, alkali may have extracted additional polysaccharide although the fact that many non-clot producers gave extracellular polysaccharides of the same composition as the clot producers supports the view that in many cases this is not so. None of the extracellular polysaccharides in Table 2 extracted with alkali are likely to be the lipopolysaccharides extracted with phenol at $60^{\circ}$ by Graham \& O'Brien (1968) as these always contained rhamnose and glucosamine.

\section{Effect of culture age on polysaccharide composition}

The fast-growing acid-producing rhizobia gave good yields of polysaccharide after 10 days incubation. In view of the much slower growth and lower polysaccharide yields of the non-acid-producing rhizobia, cultures of two of these strains (NZP 202 If and 2087) were processed for polysaccharide after 3, 6, Io and I6 days growth. The proportions of monosaccharides in these polysaccharides showed little change with growth after 3 days except that there was a gradual increase in the proportion of $X_{1}$ in 
the NZP 2087 polymer at the expense of glucose and uronic acid. Consequently it is assumed that Io day cultures yield polysaccharide representative of each non-acidproducing strain.

\section{Identity of monosaccharides}

The monosaccharides recorded as glucose, galactose, mannose, fucose and rhamnose behaved like authentic specimens on papers developed with solvents (1), (2), (3) or (4) respectively and reacted similarly with aniline hydrogen phosphate and anisidine $\mathrm{HCl}$. Unknowns $X_{1}$ and $X_{2}$ moved at clearly different rates from these latter sugars with $R$ glucose values respectively of $I \cdot 26$ and $I \cdot 62$ in solvent $(\mathrm{I})$ and $\mathrm{I} \cdot 70$ and $2 \cdot 4 \mathrm{I}$ in solvent (2). The $\mathrm{R}$ glucose values of the other sugars in these solvents were: in solvent (I), I. I2 mannose, 0.87 galactose, I.4I fucose, I.87 rhamnose; in solvent (2), I.50 mannose, 0.80 galactose, 2.20 fucose and 3.80 rhamnose. $X_{1}$ and $X_{2}$ gave the colour reactions of simple, reducing aldohexoses with aniline hydrogen phosphate, anisidine $\mathrm{HCl}$, and diphenylamine-aniline sprays. Their reactions or non-reactions with these and sprays of naphthoresorcinol, ninhydrin or thiobarbiturate indicated that they were not pentoses, ketoses, amino sugars, 2- or 6-deoxy aldohexoses or $\gamma$ or $\delta$ lactones. Rates of movement in solvents (I) and (2) and colour reactions with aniline hydrogen phosphate suggested that they were not monosaccharide uronic acid lactones.

Neither $X_{1}$ nor $X_{2}$ were fermented by yeast and treatment of the hydrolysates with yeast followed by ion-exchange resins yielded solutions free of all other sugars. When freeze-dried solids from these solutions were treated with boron trichloride (Allen, Bonner, Bourne \& Saville, 1958) $X_{1}$ and $X_{2}$ remained unchanged suggesting that they were not $O$-methyl aldohexoses and their nature remains to be established. Unknown $\mathrm{X}_{3}$ gave the colour reaction of a methyl pentose but moved clearly faster ( $\mathrm{R}$ glucose, $2 \cdot 15)$ than rhamnose ( $R$ glucose, $I \cdot 87)$ in solvent (I).

\section{Electron microscopy of organisms and associated polysaccharide}

Electron micrographs prepared directly from cultures of acid-producing (NZP 20I4, 2024) and non-acid, clot-producing (NZP 2076, 5032) strains showed extracellular, possibly mucilagenous material, associated with the bacteria. This extracellular material seemed to have a different appearance on the micrographs of the clotproducing organisms as compared with the non-clot-producing ones. Other electron micrographs showed that the acid-producing cultures gave clean organisms by simple dilution and centrifugation, whereas redispersion of the centrifuged clot from the nonacid-producing cultures gave the same electron micrograph as the original culture. After alkali treatment, however, these latter clot-producing organisms gave bacteria free from extracellular material. Micrographs prepared from cultures of non-acid-, non-clot-producing strains (NZP 2247, 2257; WU 25) showed minimal or zero amounts of extracellular material of either physical type. We believe that the electron microscope results are consistent with the extracellular material being the polysaccharide isolated in the present study.

\section{Polysaccharide composition in relation to effectiveness patterns in Lotus strains rhizobia}

Effectiveness patterns of the lotus rhizobia in nitrogen fixation with various species of Lotus and other genera (Ulex, Cytisus, Ornithopus and Lupinus) occurring in New 
Zealand are complex. A detailed study of these patterns obtained with the strains used in the present work is in progress. The following points appear relevant to polysaccharide composition.

All of our strains effective in nitrogen fixation with Lotus corniculatus L. and ineffective with $L$. pedunculatus Cav. are acid-producers, except for strains $\mathrm{CC} 1005$ and NZP 2227. These two were classed as non-acid-producers as they did not produce acid under the conditions defined by Norris (1965). Strain CC 1005 yielded no polysaccharide, a result which is in agreement with its assignment to the non-acid producers. Strain NZP 2227 gave no clot and yielded polysaccharide $(0.89 \mathrm{~g}$. $/ 1$.) showing the typical monosaccharide composition for polymer from an acid-producing strain except for a rather high mannose value. Thus this strain might be better placed within the acid-producers.

Eleven isolates included in this study formed ineffective nodules with Lotus pedunculatus, L. hispidus Desf. and L. corniculatus, but were effective with gorse (Ulex europaeus L.) and broom (Cytisus scoparius L.). Of these, six (NZP 2I4I, 2I 5 I, 5032, 5033, 5035 and 5044) gave polysaccharides of the same monosaccharide composition, including $X_{1}$, but the remaining five (NZP 2 r70, 2186, 5042, 5052 and 5144) gave a variety of different patterns in their polysaccharides, and the other strains giving $X_{1}$ all differed in their effectiveness with various host species.

Among non-acid-producing strains that are at least partially effective with Lotus pedunculatus or L. hispidus there is an apparent correlation between effectiveness with one or other of these species and the presence or absence of uronic acid in the polysaccharide, provided strains effective with both species are excluded. This is shown in Table 3 .

Table 3. Uronic acid content of polysaccharide from non-acid-producing rhizobia

\begin{tabular}{|c|c|c|c|}
\hline \multicolumn{2}{|c|}{ Effectivity on } & \multicolumn{2}{|c|}{ Number of strains giving polysaccharide with } \\
\hline $\begin{array}{l}\text { Lotus } \\
\text { pedunculatus }\end{array}$ & Lotus hispidus & High uronic acid & Low or nil uronic acid \\
\hline High & Partial or nil & $\begin{array}{l}\text { NZP 202 If, 2089, 21 } 54, \\
2181,2189,2193,2254\end{array}$ & None \\
\hline Partial & High & NZP 2192, 5074 & $\begin{array}{l}\text { NZP 2075, 2076, 2088, } \\
2175,2240,2243,5025 \text {, } \\
5091,5113\end{array}$ \\
\hline High & High & $\begin{array}{l}\text { NZP 2087, 21 83, 5087, } \\
\text { CC 814S }\end{array}$ & $\begin{array}{l}\text { NZP } 2100,2 \text { I 82, 2247, } \\
2257\end{array}$ \\
\hline Partial & Partial & $\begin{array}{l}\text { NZP } 2128,2180,2200, \\
5059 / 2,5088, \text { WU } 425\end{array}$ & $\begin{array}{l}\text { NZP 2073, 5026, 525r/2, } \\
5274\end{array}$ \\
\hline
\end{tabular}
effective with Lotus species

\section{DISCUSSION}

Individual monosaccharide values recorded in the present work may be low, particularly in high uronic acid polysaccharides, as no allowance is made for any monosaccharide present in the hydrolysate as aldobiouronic acid. The following conclusions appear to be independent of this limitation.

In general the polysaccharides from acid-producing rhizobia possess a remarkably similar monosaccharide composition except for the lack of uronic acid in poly- 
saccharides from lucerne and goat's rue rhizobia. Amager et al. (1967), investigating lucerne rhizobia, and Humphrey \& Vincent (1959) working with a wide range of acidproducing strains, found no uronic acid only in lucerne rhizobial polysaccharides. This apparent correlation may be fortuitous as Clapp \& Davis (1970) have reported a high uronic acid polysaccharide from a lucerne strain and a uronic acid-free polysaccharide from a clover rhizobium strain. It is perhaps relevant that Graham (1964), on the basis of a large number of tests not including polysaccharide composition, separated lucerne rhizobia (Rhizobium meliloti) from all other fast-growing acid-producing rhizobia he studied which he grouped under $R$. leguminosarum.

Polysaccharides produced by the non-acid-producing strains (Table 2) are, in monosaccharide composition, quite different as a class from polysaccharides produced by acid-producing rhizobial strains (Table I). Galactose is often absent (I6 out of 54), mannose is always present as a major component, while fucose, rhamnose and three unidentified probable sugars may be present. Uronic acids are also commonly absent or very low (19 out of 54). These polysaccharides also differ from those of acidproducing rhizobia in the frequent ( 36 out of 54 strains) occurrence of the polysaccharide as a diffuse mucilagenous clot after centrifuging the cultures.

Concerning rhizobial relationships within the lotus rhizobia, evidence from extracellular polysaccharides, apart from two possible exceptions, clearly supports their separation into the acid-producing and non-acid-producing groups. The acid-producing lotus rhizobia give polysaccharides not distinguishable in monosaccharide content from a number of other acid-producing rhizobial groups quite unrelated symbiotically. In the non-acid-producing rhizobia no clear distinctions could be drawn between the polysaccharides of the lotus rhizobia and the few non-lotus strains studied, both groups giving a great variety of monosaccharide patterns.

Norris (1965) suggested that non-acid-producing rhizobia represented the primitive type, and that acid-producing rhizobia were more advanced. However, the finding of close similarity of monosaccharide pattern in different cross-inoculation groups within the acid-producers, compared with the wide range of patterns in the non-acidproducers, is not easy to reconcile with Norris's suggestion of evolution of the different acid-producing groups independently from non-acid-producing rhizobia. The possibility is not excluded, however, that the acid-producers arose from the non-acidproducers and later became specialized in host range.

Thanks are due for gifts of rhizobial strains and to Mrs V. Pain, Mrs J. Ridley and Miss C. Liddane for technical assistance.

\section{REFERENCES}

$\checkmark$ Allen, S., Bonner, R. G., Bourne, E. J. \& SAVILle, N. M. (1958). Boron trichloride as a degradative reagent for carbohydrates and their derivatives. Chemistry and Industry, p. 630.

Amarger, N., Obaton, M. \& Blachère, H. (1967). Polysaccharides extracellulaires de Rhizobium meliloti. Canadian Journal of Microbiology $\mathbf{1 3}, 99-105$.

BaILEY, R. W. (1967). Loss of ingested plant carbohydrates from the reticulo-rumen. New Zealand Journal of Agricultural Research 10, 15-32.

BaileY, R. W. (1969). In Data for Biochemical Research, 2nd edn, p. 539. Edited by R. M. C. Dawson, D. C. Elliott, W. H. Elliott \& K. M. Jones. Oxford University Press.

Clapp, C. E. \& Davis, R. J. (1970). Properties of extracellular polysaccharides of Rhizobium. Soil Biology and Biochemistry 2, 109-1 17. 
DudMAN, W. F. \& HeIEELBERGER, M. (1969). Immunochemistry of newly found substituents of polysaccharides of Rhizobium species. Science, New York 164, 954-955.

Fred, E. B., Baldwin, I. L. \& McCoy, E. (1932). Root nodule bacteria and leguminous plants. University of Wisconsin Studies in Science 5, $343 \mathrm{p}$.

GrahaM, P. H. (1964). The application of computer techniques to the taxonomy of the root-nodule bacteria of legumes. Journal of General Microbiology 35, 5I I-5I7.

Graham, P. H. (1965). Extracellular polysaccharides of the genus Rhizobium. Antonie van Leeuwenhoek 3I, 349-354.

Graham, P. H. \& O'Brien, M. A. (1968). Composition of lipopolysaccharides from Rhizobium and Agrobacterium. Antonie van Leeuwenhoek 34, 326-330.

HumphreY, B. A. \& VINCENT, J. M. (1959). Extracellular polysaccharides of Rhizobium. Journal of General Microbiology 21, 477-484.

Montreuil, J. \& SpIK, G. (I963). Microdosage des Glucides, vol. I, p. 6o. France: Faculté des Sciences de Lille.

NoRris, D. O. (1965). Acid production of Rhizobium. A unifying concept. Plant and Soil 22, 143-166.

WiLson, C. M. (1959). Quantitative determination of sugars on paper chromatograms. Analytical Chemistry 3r, I I99-120r. 